\title{
REACTIVE MOLECULAR DYNAMICS SIMULATION AND CHEMICAL KINETIC EVALUATION OF COMBUSTION OF TRIETHYLALUMINIUM (TEA)
}

\author{
Rene F. B. Gonçalves, Koshun Iha and José A. F. F. Rocco*
}

Departamento de Química, Instituto Tecnológico de Aeronáutica, 12228-900 São José dos Campos - SP, Brasil

Recebido em 02/10/2017; aceito em 17/01/2018; publicado na web em 22/02/2018

\begin{abstract}
The combustion process of triethylaluminum is investigated by means of reactive molecular dynamics simulations using the ReaxFF force field. The behavior of the system in five different temperatures ranging from 2000-4000 K was evaluated. As a pyrophoric material, TEA reacts also with water, generating gaseous hydrogen, whose content increases with the system temperature. Rapid water formation and $\mathrm{O}_{2}$ depletion were observed and, using Arrhenius equation, the preexponential factor and activation energy were found to be $9.67 \mathrm{E}+09 \mathrm{~s}^{-1}$ and $1.242 \mathrm{~kJ} \mathrm{~mol}^{-1}$, respectively. The results obtained are in accordance to the expected for pyrophoric materials and the simulation in question can help elucidating and analyzing the complex reaction mechanism of TEA combustion.
\end{abstract}

Keywords: triethylaluminium (TEA); combustion; ReaxFF; reactive molecular dynamics simulation.

\section{INTRODUCTION}

The use of flares in military aircrafts became almost indispensable for protection against infrared (IR) missiles. ${ }^{1}$ A missile approach warning system carried by the aircrafts detects a launched missile and then release IR flares as decoys. This flare gives the IR seeker a more attractive IR emitting source to track, instead of the original target. ${ }^{2}$

Flares are composed mainly by pyrophoric reagents, i.e. substances that self-ignite in air at temperatures of $54.4{ }^{\circ} \mathrm{C}$ or less. ${ }^{3}$ Oxidation of the pyrophoric reagents by oxygen or exothermic reactions with moisture in the air (resulting in the generation of a flammable gas such as hydrogen) is so rapid that ignition occurs spontaneously.

Pyrophorics are also used to shorten the ignition delay time and to increase the energy density of fuels. Pyrophoric organometallic compounds may also provide an ignition source and flame stabilizing mechanism within the combustor, thus permitting use of hydrocarbon fuels in supersonic combustion systems. Triethylaluminum (TEA) is one of the materials used for this application due to their high energy density and reactivity. ${ }^{4}$

Combustion of these materials is usually difficult to analyze experimentally due to their high reactivity and safety measures required. A great effort is needed for elucidating the mechanisms and evaluating the rates and intermediate species. Consequently, kinetic modeling plays a critical role in uncovering the complex reaction mechanisms. Reactive Molecular Dynamics (RMD) simulations is one of the solutions for this problem, as it requires low computational cost and provides accurate prediction of the interactions among the species present in the simulation boundaries. ReaxFF ${ }^{5}$ is a force field developed for simulation of chemical reactions, where bonds are broken and formed several times during the simulation. This method has been extensively used for combustion of energetic materials, ${ }^{6,7}$ thermal decomposition of polymers, fuel cells, catalysts ${ }^{8-10}$ and so on.

In the present work, RMD simulations were performed for the reaction between triethylaluminum and gaseous oxygen in a fuel rich condition, in five different temperatures from 2000 to $4000 \mathrm{~K}$. A kinetic analysis of the global process is also developed with the simulation results.

*e-mail: renefbg@ita.br

\section{SIMULATION DETAILS}

The reactive molecular dynamics simulations were performed using the software LAMMPS (Large-scale Atomic/Molecular Massively Parallel Simulator) and the ReaxFF5 force field. In this force field, the general energy function takes the following formulation:

$\mathrm{E}_{\text {system }}=\mathrm{E}_{\mathrm{bon}}+\mathrm{E}_{\text {over }}+\mathrm{E}_{\text {under }}+\mathrm{E}_{\mathrm{val}}+\mathrm{E}_{\mathrm{pen}}+\mathrm{E}_{\text {tors }}+\mathrm{E}_{\text {conj }}+\mathrm{E}_{\mathrm{vdWaal}} \mathrm{s}+\mathrm{E}_{\text {Coulomb }}$

which:

- Ebond represents the bond energy;

- $E_{\text {over }}$ and $E_{\text {under }}$ denotes the over- and under-coordinated atom in the energy contribution, respectively;

- $E_{\text {val }}, E_{\text {pen }}, E_{\text {tors }}$ sare the valence angle term, penalty energy and torsion energy, respectively;

- $E_{\text {coni }}, E_{\text {vdWaals }}, E_{\text {Coulomb }}$ represent the conjugation effects to molecular energy, nonbonded van der Waals interaction and Coulomb interaction, respectively.

ReaxFF has a fundamental assumption where the bond order and bond energy between a pair of atoms is obtained from the interatomic distance, according to the equation below. In this equation is possible to observe the contribution of the sigma bond $\left(\mathrm{p}_{\mathrm{bo}, 1}\right.$ and $\left.\mathrm{p}_{\mathrm{bo}, 2}\right)$, the first pi bond $\left(\mathrm{p}_{\mathrm{bo}, 3}\right.$ and $\left.\mathrm{p}_{\mathrm{bo}, 4}\right)$ and the second pi bond $\left(\mathrm{p}_{\mathrm{bo}, 5}\right.$ and $\left.\mathrm{p}_{\mathrm{bo}, 6}\right)$.

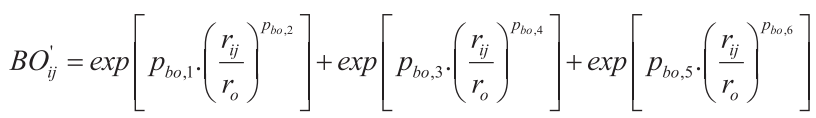

The bond orders $\mathrm{BO}_{\mathrm{ij}}^{\prime}$ are then corrected for overcoordination and for residual 1-3 bond orders in valence angles. A corrected bond order $\left(\mathrm{BO}_{\mathrm{ij}}\right)$ is used to calculate the bond energies:

$$
E_{b o n d}=-D_{e} \cdot B O_{i j} \cdot \exp \left[p_{b e, 1} \cdot\left(1-B O_{i j}^{p_{b e, 1}}\right)\right]
$$

Besides these terms, ReaxFF also takes into account over and undercoordination, valence angle terms (energy contribution from valence angle terms should go to zero when $\mathrm{BO}$ is zero), torsion angles (for both $\mathrm{BO} \rightarrow 0$ and $\mathrm{BO}>1$ ), conjugation effects to the molecular energy, nonbonded van der Waals interactions (repulsive interactions at short interatomic distances due to Pauli principle orthogonalization 
and attraction energies at long distances due to dispersion, for all atom pais), Coulomb interactions (for all atoms, adjusted for orbital overlap between atoms at close distances with a shielded Coulomb potential).

The force fields used in ReaxFF were optimized using a successive one-parameter search technique, where data obtained from quantum chemical calculations (DFT) were used. Heats of formation to within $4.0 \mathrm{kcal} / \mathrm{mol}$ were reproduced using this technique.

At every MD step, this force field updates the bond orders and provides a pathway for bonds to form and break during the course of the simulation. ${ }^{8}$ ReaxFF can reproduce with acuity all relevant quantum mechanical data, as well as provide atomistic descriptions of several complex chemical reactions. ${ }^{11}$ In this work, RMD simulations results are also employed for the calculation of the Arrhenius parameters for the global reaction.

The combustion simulation was done using the condensed phase structure of triethylaluminum in a fuel rich condition (less than stoichiometric quantity of oxygen molecules), in five different temperatures $(2000 \mathrm{~K}, 2500 \mathrm{~K}, 3000 \mathrm{~K}, 3500 \mathrm{~K}$ and $4000 \mathrm{~K})$. Three dimers of trietylaluminum were inserted in a unit cell measuring 12.4 × 14.4 × $6.9 \AA$, generating a condensed phase structure with approximate density of $0.92 \mathrm{~g} \mathrm{~cm}^{-3}$ (experimental density: $0.832 \mathrm{~g} \mathrm{~cm}^{-3}$ ). This structure was put in contact with 30 molecules of gaseous oxygen inside a box of 20 x 20 × $20 \AA$, where the collisions/ reactions can occur. The pressure at the beginning of the simulation was calculated using the ideal gas equation of state for the chosen temperatures and can be seen in Figure 1. The high pressure and temperatures used in the simulations facilitates the collisions among molecules, thus decreasing the necessary time and computational costs of the calculations.

The system was minimized using low temperature $(5 \mathrm{~K})$ molecular dynamics with constant number of atoms $(\mathrm{N})$, constant volume (V) and control of the potential energy (E), denoted as NVE. After minimization, for the production phase, the NVT ensemble was used. As reactive molecular dynamics involve usually fast reactions, a timestep of 0.1 femtoseconds (fs) was used. The total simulation time was $100 \mathrm{ps}$ for each of the selected temperatures. The temperatures were controlled by the Berendsen thermostat, with temperature damping constant of $100 \mathrm{fs}$.

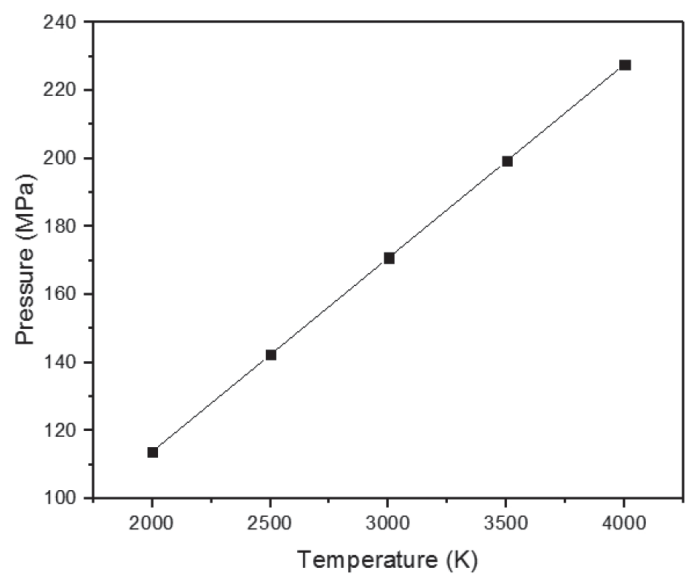

Figure 1. Pressure at the beginning of the RMD simulations

For the kinetic analysis of the TEA combustion, apparent firstorder kinetics was used to describe the global reaction. The oxygen content was considered for calculating the rate constant $(\mathrm{k})$, according to the linear fitting of the number of oxygen molecules $(\mathrm{N})$ against the simulation time $(\mathrm{t})$ :
The fitting parameters obtained from this relation enables the calculation of the activation energy and the frequency factor through the Arrhenius equation.

\section{RESULTS AND DISCUSSION}

In early steps, an initial fragmentation of TEA is achieved, i.e. after approximately $2-5 \mathrm{ps}$, there is abstraction of hydrogen atoms by the oxygen molecules, generating $\mathrm{HO}_{2}$, and even the formation of $\mathrm{H}_{2}$ and water molecules. As the system is kept under high pressure and temperatures, the collisions are more effective and cause breakages of external bonds. During the simulation, fragments of $\mathrm{C}_{2} \mathrm{H}_{4}, \mathrm{C}_{2} \mathrm{H}_{6}$ and $\mathrm{C}_{2} \mathrm{H}_{2}$ are produced. These fragments appear due to the Al- $\mathrm{C}$ bond break, possibly due to strong collisions of the gas particles or even the other fragments present in the system.

The evolution of the species generated from the combustion of the TEA molecules are shown in Figure 2. The increasing temperature has a positive effect on the reaction velocity, as the water content increases with the temperature. The shape of the curves, i.e. the behavior of the system is different in each scenario, as the temperature levels can enable different elementary processes. In higher temperatures, the slope of the water formation curves increases significantly, which can be translated in the previously mentioned velocity increase.

In the total time considered for the simulations, the carbon dioxide content was still low compared to water. Carbon monoxide, however, was generated in larger scale as the temperature was higher. The fuel rich condition was selected due to the application of TEA as pyrophoric flare, and in high altitudes, the oxygen availability decreases, and the combustion process becomes incomplete. Therefore, it is expected to have a higher content of carbon monoxide in the system. The total combustion process should than take much longer time ( $>100 \mathrm{ps})$ to occur, in the simulated conditions.

One of the most important parameters for evaluating the evolution and efficiency of a combustion process regards the complete products formation (carbon dioxide and water). As previously stated, the combustion was simulated under a fuel rich condition. Therefore, is expected to have a larger number of water molecules, as the availability and stereo factor of the hydrogen atoms in TEA is much larger than the carbon ones. Also, for being able to produce $\mathrm{CO}_{2}$, the Al-C bonds must be broken, producing $\mathrm{C}_{2} \mathrm{H}_{\mathrm{x}}$ fragments. These fragments suffer oxidation, producing water, carbon monoxide and carbon dioxide, which is possible if there are enough oxidizer species.

TEA is a known pyrophoric material, i.e. reacts (burns) instantly in the presence of oxygen. As observed in the simulations, all TEA species starts to fragment after 4-5 ps; the first observed reaction in the abstraction of hydrogen by the $\mathrm{O}_{2}$ and also the production of $\mathrm{C}_{\mathrm{x}} \mathrm{H}_{\mathrm{y}}$ species, from the decomposition of the ethyl ramifications. These bonds are mostly broken due to the collisions of the gaseous species present in the mixture.

It is interesting to observe the evolution of the fragments (in terms of different chemical species) in the system, according to the temperature (Figure 3). As expected, higher temperatures lead to increasing fragment production, especially in the first decomposition steps. However, as the reaction proceeds, the quantity of fragments varies significantly and differently in each temperature, due to the complex kinetic mechanism and activation energies of each step. At the end of the simulation, the system kept at $2000 \mathrm{~K}$ was the one with the largest number of fragments. Most of these fragments are intermediate species, which shall be consumed to generate the final combustion products posteriorly. At $4000 \mathrm{~K}$, several intermediates have been consumed, and more final product species are present, reducing the final number of different chemical species in the cell. 

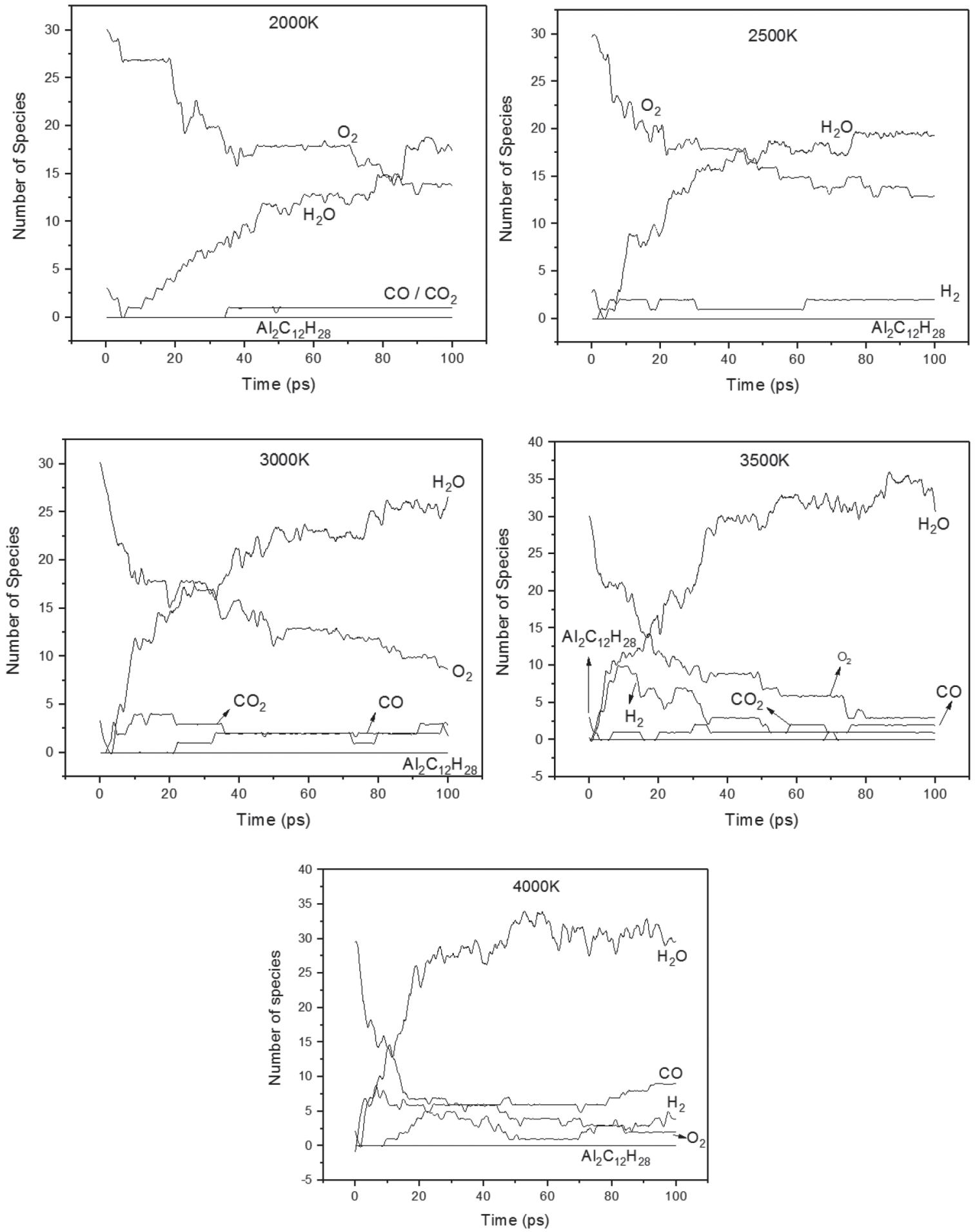

Figure 2. Evolution of the generated species in each temperature condition

Figure 4 presents the variation of the number of water molecules, showing the same behavior, i.e. the initial production rate of the species is larger at higher temperatures and after 20-30 ps varies according to the combustion mechanism (different elementary reactions activated/possible in each temperature).

The very presence of moisture around pyrophoric reactants has an effect of flammable gas generation (usually $\mathrm{H}_{2}$ ). As TEA is a known pyrophoric agent, the production of gaseous hydrogen would be expected, as there is fast water formation during the combustion. Figure 5 shows the variation of $\mathrm{H} 2$ content in the simulations.
Especially in higher temperatures, the number of $\mathrm{H}_{2}$ molecules produced is increased. As a highly flammable gas, the hydrogen produced in TEA combustion increases the overall heating rate and accelerates the burning of the material, due to its high reactivity and reaction velocity.

A proposed pathway is observed in Scheme 1 below. This mechanism is proposed based on the species formation and decomposition, observed in the simulations. As there is currently no mechanism in the literature for the TEA combustion, the reactions presented may be useful for a preliminary analysis and as an initial development for a detailed kinetic mechanism. 


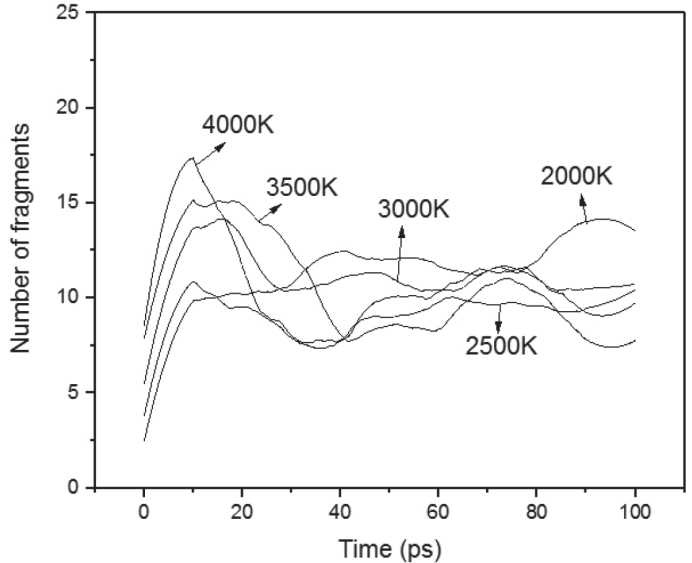

Figure 3. Variation of the number of fragments according to the temperature of the system

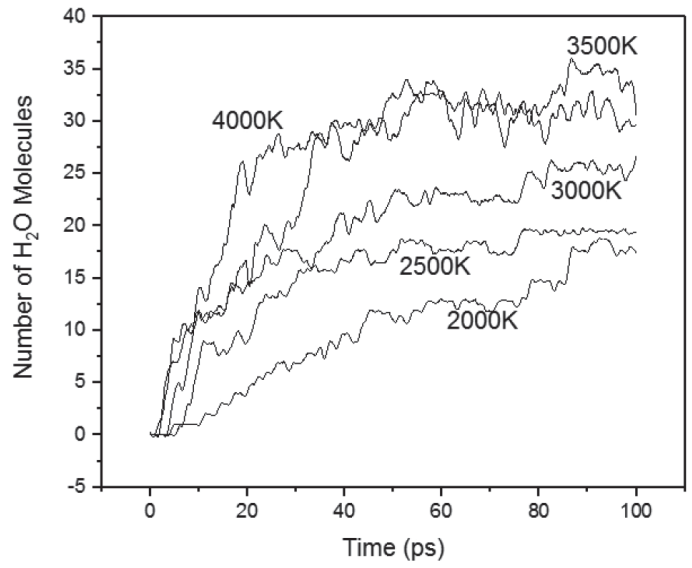

Figure 4. Comparison of the water formation in each scenario

Similar to $\mathrm{H}_{2} \mathrm{O}$, the oxygen content analysis can provide information on the progress of the combustion process. In this case (Figure 6a) a kinetic analysis was possible when considering the overall combustion of TEA a first-order reaction, so the number of $\mathrm{O}_{2}$ molecules at each time $\mathrm{t}$ was compared to the initial number $\left(\mathrm{N}_{0}\right)$ inside the box. Doing so, each temperature provided a different value of the rate constant $(\mathrm{k})$, which can be plotted against the inverse temperature (Figure $6 \mathrm{~b}$ ). The slope of the obtained line is used for calculating the global activation energy (EA), and the y-interception leads to the value of the frequency factor (A). This behavior follows Arrhenius equation:

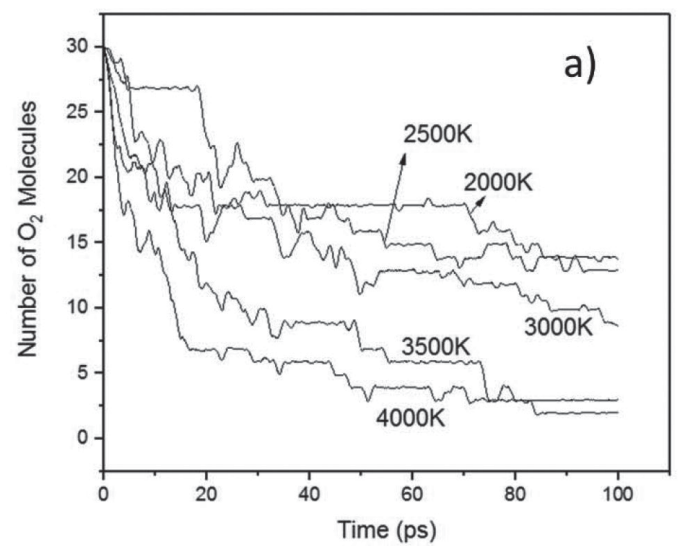

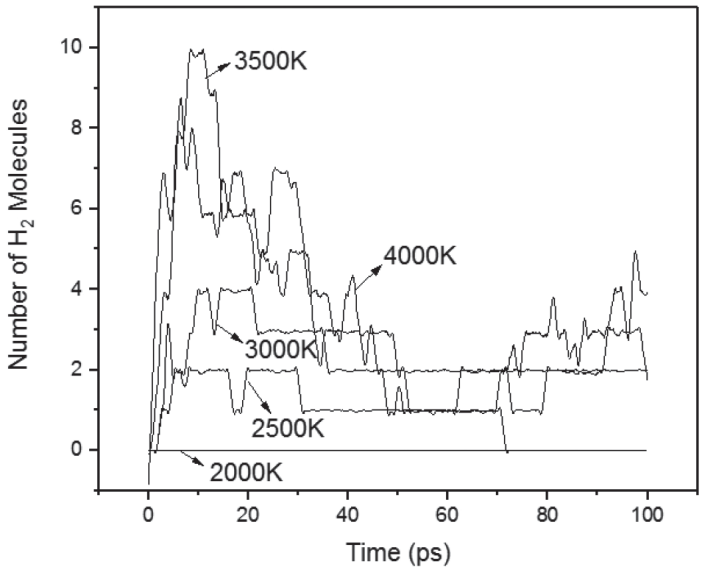

Figure 5. Analysis of $\mathrm{H}_{2}$ production from contact between moisture and pyrophoric substance (TEA)

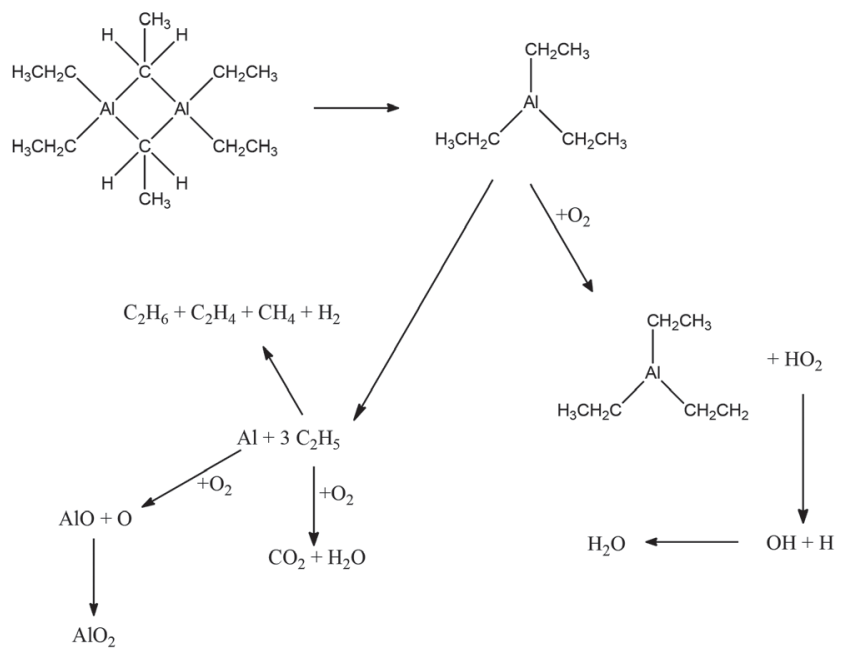

Scheme 1. Proposed main pathways for TEA combustion

$$
k=A \cdot e^{\left(\frac{-E_{A}}{R T}\right)}
$$

or, in its linear form:

$$
\ln k=\ln A-\frac{E_{A}}{R} \cdot \frac{1}{T}
$$

As expected from the Arrhenius equation $(\ln \mathrm{k} \propto 1 / \mathrm{T})$, the rate constant decreases linearly as the temperature increases, with a

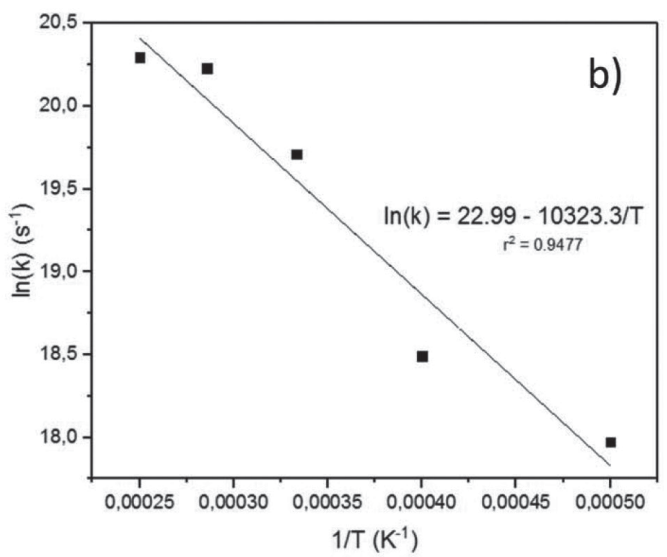

Figure 6. a) Oxygen molecules consumption b) Arrhenius plot of TEA at the five temperatures 
correlation of $95 \%$ among the obtained values from the RMD simulation.

Using the linear equation obtained from the plot, the values of activation energy and frequency factor may be seen below:

$$
\begin{array}{ll}
\mathrm{Ea}(\mathrm{kJ} / \mathrm{mol}) & 1.242 \\
\mathrm{~A}\left(\mathrm{~s}^{-1}\right) & 9.67 \mathrm{E}+09
\end{array}
$$

A very low activation energy was obtained, which is highly expected for a pyrophoric material. This indicates that the reaction between TEA and $\mathrm{O}_{2}$ is spontaneous. For comparison purposes, the experimental flashpoint of TEA is $-52.78{ }^{\circ} \mathrm{C}$.

Figure 7 shows the time evolution of the potential energies during the NVE-MD simulations. The potential energies decrease as the reactions occurred during MD simulations. Especially at high temperatures, the potential energies decrease very quickly. However, as the molecular simulation moves on, the potential energies show no obvious changes. The number of oxygen molecules consumed in the oxidation processes of TEA molecules increase rapidly as the temperature goes beyond $2000 \mathrm{~K}$. The potential energies show nearly the same tendency as the number of oxygen molecules, which suggested that the oxidation reactions are exothermic, and this lead to the decrease of the total potential energies.

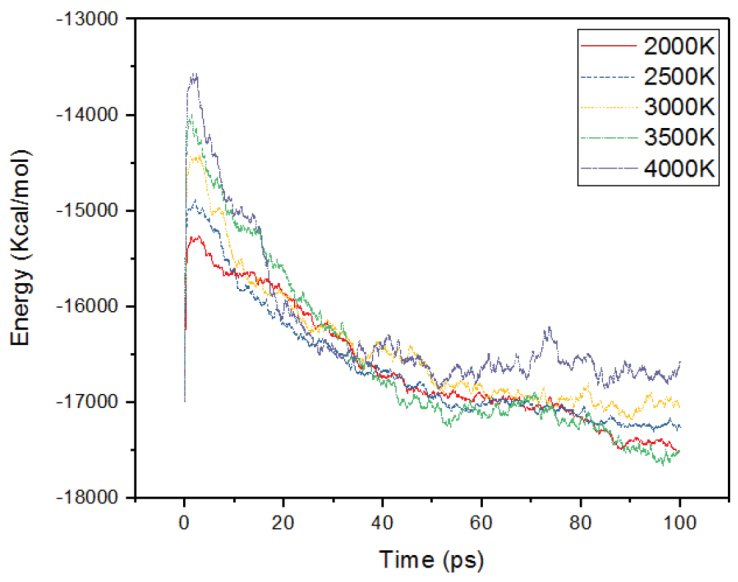

Figure 7. Potential energies during NVE-MD simulations of TEA at different temperatures

\section{CONCLUSION}

The reactive force field, ReaxFF, is applied to investigate the combustion of TEA. The initiation mechanism of the combustion involves mainly the abstraction of hydrogen atoms from the ethyl groups and then the rupture of $\mathrm{Al}-\mathrm{C}$ bonds, leading to the formation of small hydrocarbon species and $\mathrm{CO}_{2}$ in the end. Apparent firstorder kinetics is used to describe the combustion of TEA, and the prediction of the activation energy and frequency factor are in accordance to the pyrophoric characteristic of the material. The results obtained indicate that ReaxFF can be used to predict the behavior of fast combustion processes and also assist the construction and optimization of molecular based kinetic reaction mechanisms used for chemical kinetic modeling.

\section{ACKNOWLEDGEMENT}

Research developed with the assistance of CENAPAD-SP (National Center for High Performance Processing in São Paulo), project UNICAMP / FINEP - MCT.

\section{REFERENCES}

1. Birchenall, R. P.; Richardson, M. A.; Butters, B.; Walmsley, R.; Infrared Phys. Technol. 2011, 54, 412.

2. Jackman, J.; Richardson, M.; Butters, B.; Walmsley, R.; Yuen, P.; James, D.; Infrared Phys. Technol. 2011, 54, 121.

3. Alnajjar, M.; Quigley, D.; Kuntamukkula, M.; Simmons, F.; Freshwater, D.; Bigger, S.; J. Chem. Heal. Saf. 2011, 18, 5.

4. Ryan, T. W.; Schwab, S. T.; Harlowe, W. W.; Langley Research Center; Ignition delays, heats of combustion, and reaction rates of aluminum alkyl derivatives used as ignition and combustion enhancers for supersonic combustion, 1992. Hampton, Va., National Aeronautics and Space Administration, Langley Research Center; National Technical Information Service, distributor.

5. van Duin, A. C. T.; Dasgupta, S.; Lorant, F.; Goddard, W. A.; J. Phys. Chem. A 2001, 105, 9396.

6. Manaa, M. R.; Fried, L. E.; Melius, C. F.; Elstner, M.; Frauenheim, T.; J. Phys. Chem. A 2002, 106, 9024.

7. Yanagihara, H.; Stanković, I.; Blomgren, F.; Rosén, A.; Sakata, I.; Combust. Flame 2014, 161, 541.

8. Ludwig, J.; Vlachos, D. G.; van Duin, A. C. T.; Goddard, W. A.; J. Phys. Chem. B 2006, 110, 4274

9. Strachan, A.; van Duin, A. C. T.; Chakraborty, D.; Dasgupta, S.; Goddard, W. A.; Phys. Rev. Lett. 2003, 91, 98301.

10. Hong, S.; Van Duin, A. C. T.; J. Phys. Chem. C 2016, 120, 9464.

11. Wang, Q.-D.; Wang, J.-B.; Li, J.-Q.; Tan, N.-X.; Li, X.-Y.; Combust. Flame 2011, 158, 217. 